\title{
Erratum: Fresh from the pipeline 2011
}

Jim Kling

Nat. Biotechnol. 30, 128-131 (2012); published online 8 February 2012; corrected after print 8 February 2012

In the print version of this article, the title reads "Fresh from the pipeline 2011." It should read "Fresh from the biotech pipeline-2011. The title has been corrected in the HTML and PDF versions of the article before online publication.

\section{Erratum: Profiling PARP inhibitors}

Philip Jones

Nat. Biotechnol. 30, 249-250 (2012); published online 7 March 2012; corrected after print 7 March 2012

In the print version of this article, MD Anderson is listed as Maryland Anderson. The error has been corrected in the HTML and PDF versions of the article before online publication.

\section{Corrigendum: The BioPAX community standard for pathway data sharing}

Emek Demir, Michael P Cary, Suzanne Paley, Ken Fukuda, Christian Lemer, Imre Vastrik, Guanming Wu, Peter D’Eustachio, Carl Schaefer, Joanne Luciano, Frank Schacherer, Irma Martinez-Flores, Zhenjun Hu, Veronica Jimenez-Jacinto, Geeta Joshi-Tope, Kumaran Kandasamy, Alejandra C Lopez-Fuentes, Huaiyu Mi, Elgar Pichler, Igor Rodchenkov, Andrea Splendiani, Sasha Tkachev, Jeremy Zucker, Gopal Gopinath, Harsha Rajasimha, Ranjani Ramakrishnan, Imran Shah, Mustafa Syed, Nadia Anwar, Özgün Babur, Michael Blinov, Erik Brauner, Dan Corwin, Sylva Donaldson, Frank Gibbons, Robert Goldberg, Peter Hornbeck, Augustin Luna, Peter Murray-Rust, Eric Neumann, Oliver Reubenacker, Matthias Samwald, Martijn van Iersel, Sarala Wimalaratne, Keith Allen, Burk Braun, Michelle Whirl-Carrillo, Kei-Hoi Cheung, Kam Dahlquist, Andrew Finney, Marc Gillespie, Elizabeth Glass, Li Gong, Robin Haw, Michael Honig, Olivier Hubaut, David Kane, Shiva Krupa, Martina Kutmon, Julie Leonard, Debbie Marks, David Merberg, Victoria Petri, Alex Pico, Dean Ravenscroft, Liya Ren, Nigam Shah, Margot Sunshine, Rebecca Tang, Ryan Whaley, Stan Letovksy, Kenneth H Buetow, Andrey Rzhetsky, Vincent Schachter, Bruno S Sobral, Ugur Dogrusoz, Shannon McWeeney, Mirit Aladjem, Ewan Birney, Julio Collado-Vides, Susumu Goto, Michael Hucka, Nicolas Le Novère, Natalia Maltsev, Akhilesh Pandey, Paul Thomas, Edgar Wingender, Peter D Karp, Chris Sander \& Gary D Bader

Nat. Biotechnol. 28, 935-942 (2010); published online 9 September 2011; corrected after print 7 December 2010 and 10 April 2012

In the version of this article initially published, Oliver Reubenacker should have been spelled Oliver Ruebenacker. In addition, the location of the author's affiliation is Farmington, not Storrs. The errors have been corrected in the HTML and PDF versions of the article. 